\title{
Identification of previously untypable RD cell line isolates and detection of EV-A71 genotype C1 in a child with AFP in Nigeria
}

\author{
Olubusuyi Moses Adewumi ${ }^{a}$, Temitope Oluwasegun Cephas Faleye ${ }^{\mathrm{a}, \mathrm{b}}$, Christopher Olaoluwa Okeowo $\mathbb{D}^{c}$, \\ Akintunde Michael Oladapoc, Joyce Oyathelemhic, Olawumi A Olaniyic, Oluwatoyosi Catherine Isolac \\ and Johnson Adekunle Adeniji ${ }^{\mathrm{a}, \mathrm{d}}$
}

aDepartment of Virology, College of Medicine, University of Ibadan, Ibadan, Nigeria; 'bepartment of Microbiology, Faculty of Science, Ekiti State University, Ado-Ekiti, Nigeria; 'Department of Science Laboratory Technology, Faculty of Science, Ekiti State University, Ado-

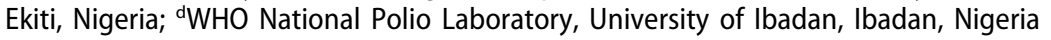

\section{ABSTRACT}

We previously attempted to identify 96 nonpolio enteroviruses (EVs) recovered in RD cell culture from children $<15$ years with acute flaccid paralysis in Nigeria. We succeeded in identifying 69 of the isolates. Here, we describe an attempt to identify the remaining 27 isolates. Twenty-six (the 27 th isolate was exhausted) isolates/samples that could not be typed previously were further analyzed. All were subjected to RNA extraction, CDNA synthesis, enterovirus 5'-UTR-VP2 PCR assay and a modified VP1 snPCR assay. Both the 5'-UTR-VP2 and VP1 amplicons were sequenced, isolates identified and subjected to phylogenetic analysis.Twenty of the 26 samples analyzed were identified. Altogether, 23 (three samples had co-infection) EV strains were recovered. These belong to 11 EV (one EVA, nine EVB and one EVC) types which were EVA71 genotype C1 (1 strain), CVB3 (7 strains), CVB5 (1 strain), E5 (2 strain), E11 (3 strains), E13 (2 strain), E19 (1 strain), E20 (1 strain), E24 (2 strains), EVB75 (1 strain) and EVC99 (2 strains). Of the 11 EV types, the 5'-UTR-VP2 assay identified seven while the VP1 assay identified 10. Both assays simultaneously detected 7 of the 11 EV types identified in this study with $100 \%$ congruence. We successfully identified 20 of 26 samples that were previously untypable. We also provided evidence that suggests a clade of EVA71 genotype C1 might have been circulating in sub-Saharan Africa since 2008. Finally, we showed that the 5'-UTR -VP2 assay might be as valuable as the VP1 assay in EV identification.
KEYWORDS

Acute Flaccid Paralysis; enteroviruses; Nigeria; $5^{\prime}$-VP2 assay; VP1 analysis

\section{Introduction}

Enteroviruses are members of the genus Enterovirus in the Picornaviridae family. Within the genus are 15 species and over 100 serotypes distributed into the different species [1]. The best studied members of the genus are the polioviruses (PVs) which are serotypes (1,2 and 3 ) in Species C. Enteroviruses have an $\sim 7.5 \mathrm{~kb}$, positivesense, single stranded RNA genome with a single open reading frame (ORF). The ORF is flanked on both sides by untranslated regions (UTRs). The 5'-UTR has subregions that are conserved among all enterovirus types hence it's being used in panenterovirus detection assays $[2,3]$. The 3'-UTR, on the other hand, has sub-regions that are conserved within species and is consequently used for EV species determination [4]. Determination of EV serological types were done using neutralization assays $[2,3,5]$. However, since a correlation was established between serological types of enteroviruses and the sequences of the VP1 gene [5], VP1 sequence analysis has been used for enterovirus identification.

In most developing nations globally, Poliovirus surveillance is the only enterovirus surveillance system in existence. Surveillance for PV entails looking for the virus in sewage and in children presenting with Acute Flaccid Paralysis (AFP) [6]. In line with the WHO recommendation, the global poliovirus surveillance programme uses $\mathrm{RD}$ (from Human Rhabdomyosarcoma [7]) and L20b (Mouse $L$ cell line genetically modified to express the human poliovirus receptor $[8,9])$ cell lines for poliovirus isolation $[3,4,6]$. While L20b cell line seems to be more specific for the polioviruses, many non-polio enteroviruses (NPEVs) also replicate in RD cell line $[3,4,6]$. Consequently, the global PV surveillance programme, generates as a byproduct several NPEVs, which till date provide most of the information that exist on NPEV diversity.

In Nigeria, there have been two studies $[10,11]$ that have investigated EV genetic diversity in AFP cases. While the first study [10] investigated samples dating from 2002 to 2004, the second [11] investigated samples collected in 2014 (about a decade after the first study). In our 2014 study [11], there were 27 isolates recovered on RD cell line from AFP cases that we could not determine their serotypes. This study was consequently designed to use alternative strategies to determine the serotypes of these previously untypables isolates. 


\section{Materials and methods}

\section{Samples}

The 27 isolates (subsequently referred to as samples) that remained untypable from our previous study [11] were the subject of this study. However, because, one of the samples was already exhausted, it could not be further analyzed. Hence, only the remaining 26 samples were further analyzed. To be precise, these 26 samples were isolates recovered on RD cell line from children ( $<15$ years old) presenting with AFP in Nigeria in 2014 [11]. The samples had been previously subjected to three enterovirus screens (Figure 1 and [11]) but, their respective types remained to be determined. It should be noted that between the time our previous [11] and this study were done, the samples were stored at $-20^{\circ} \mathrm{C}$.

\section{RNA extraction and CDNA synthesis}

All samples were re-subjected to RNA extraction using the Total RNA extraction kit (Jena Bioscience, Jena, Germany) as instructed by the manufacturer. Subsequently, cDNA was made using the SCRIPT CDNA synthesis kit (Jena Bioscience, Jena, Germany) following manufacturer's instructions with slight modifications. Two (2) separate CDNA sets were made (Figure 1). Rather than use Random Hexamers as described in [11], Primers AN32 - AN35 were used for synthesis of
CDNA 1 as previously described $[6,12,13]$ while primer OL68 was used for synthesis of CDNA 2 [14].

\section{Polymerase chain reaction (PCR) assays}

Two different PCR assays were done in this study. Assay 1 amplified a $750 \mathrm{bp}$ amplicon spanning part of the 5'-UTR through VP4 to the 5'-end of VP2 (5'UTR - VP2). Primers EVP-2 and OL68 [14] alongside CDNA two were used for this assay.

The second PCR assay (assay 2) is the modified version of the Nix et al. [12], semi-nested PCR assay described in Adeniji et al. [15]. The first round PCR amplifies a $\sim 750 \mathrm{bp}$ amplicon spanning parts of VP3 and VP1 (VP3 - VP1). Primers 224 and 222 [12] alongside CDNA1 were used for this assay. Three second round PCR assays, each amplifying an $\sim 350 \mathrm{bp}$ amplicon within the $5^{\prime}$ part of VP1 were done as described in Adeniji et al. [15]. The three second round assays ( $\mathrm{PE}, \mathrm{EV}-\mathrm{A} / \mathrm{C}$ and $\mathrm{EV}-\mathrm{B}$ ) used forward primers AN89, 189 and 187, respectively, paired with same reverse primer AN88. Three microlitres $(3 \mu \mathrm{L})$ of the product of first round PCR assay was used as template for all the three second round assays.

Thermal cycling was done using a Veriti Thermal cycler (Applied Biosystems, California, USA). For assay one and first round of assay two, cycling conditions were $94^{\circ} \mathrm{C}$ for 3 minutes, then 45 cycles of $94^{\circ} \mathrm{C}$ for 30 seconds, $42^{\circ} \mathrm{C}$ for 30 seconds, and $60^{\circ} \mathrm{C}$ for

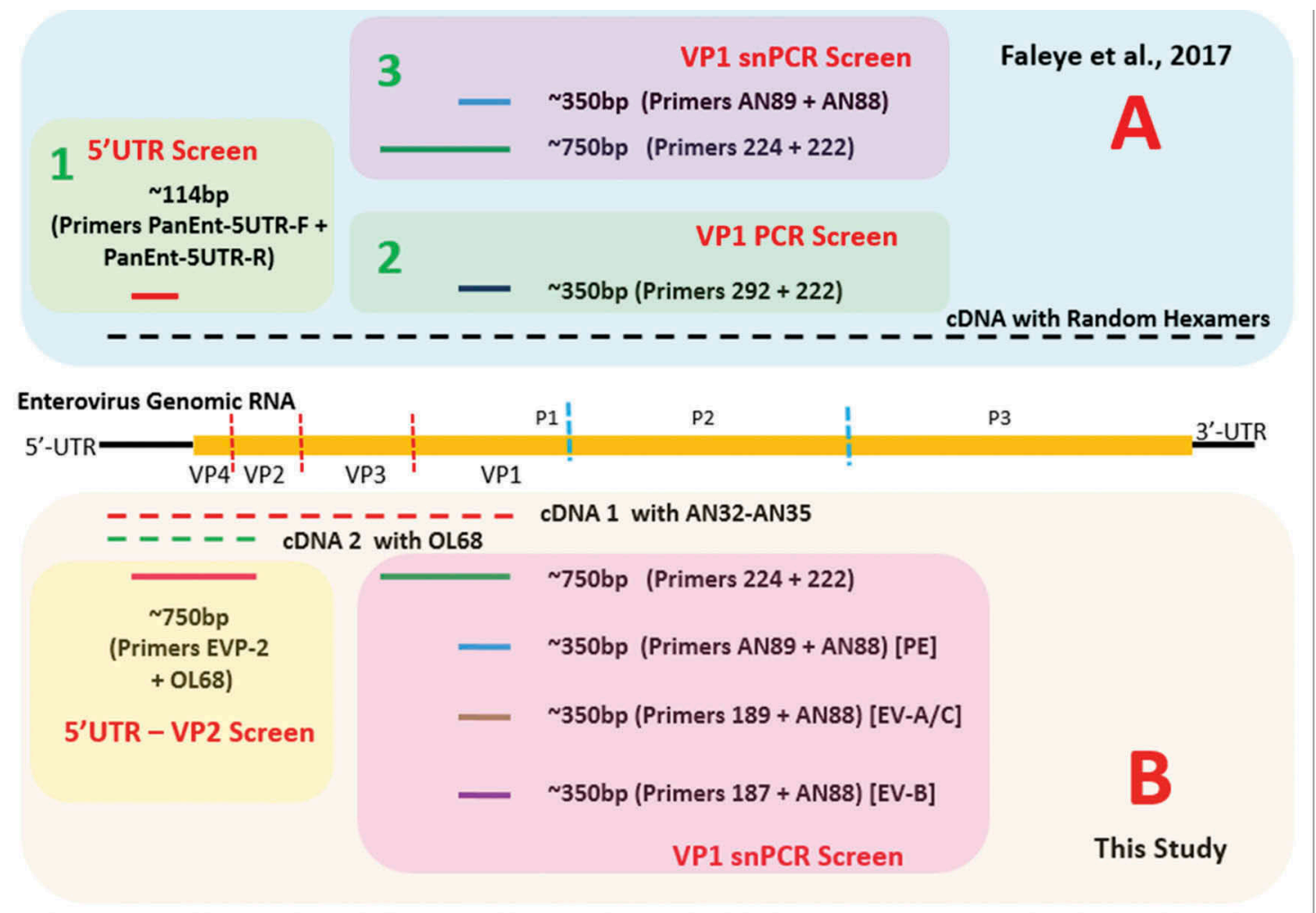

Figure 1. The algorithm followed in this study. Section ' $A$ ' of the figure depicts the screens the isolates were previously subjected to in Faleye et al., 2017 [11]. Section 'B' shows the alternative approach deployed for identifying the isolates in this study. 
60 seconds, with ramp of $40 \%$ from $42^{\circ} \mathrm{C}$ to $60^{\circ} \mathrm{C}$. This was then followed by $72^{\circ} \mathrm{C}$ for 7 minutes and held at $4^{\circ} \mathrm{C}$ until the reaction was terminated. Cycling conditions for second round of assay two were similar to that of the first round except for the extension time that was reduced to 30 seconds. PCR products of assay 1 and the second round PCR product of assay 2 were resolved on a $2 \%$ Agarose gel stained with ethidium bromide and viewed by ultraviolet (UV) light on a transilluminator.

\section{Sequencing and identification}

All amplicons of PCR reactions with the expected band size were shipped to Macrogen Inc, Seoul, South Korea, where amplicon purification and sequencing were done. Sequencing was performed using the forward and reverse primers for each of the respective assays. Subsequently, the sequence data was used for enterovirus genotype and species determination. For assay 1 the sequence data was subjected to the NCBI BLASTn tool while the sequence data for the second round PCR product of assay 2 were subjected to the enterovirus genotyping tool [16].

\section{Phylogenetic analysis}

Using the default settings of the CLUSTAL W program in MEGA 5 software [17] sequences of the EVA71 described in this study were aligned alongside those retrieved from GenBank. Afterwards a neighborjoining tree was constructed using the same MEGA 5 software with the Kimura-2 parameter model [18] and 1,000 bootstrap replicates.

\section{Nucleotide sequence accession numbers}

All the sequences reported in this study have been deposited in GenBank and assigned accession numbers MH379115-MH379135 and MH397260-MH397268.

\section{Results}

\section{PCR assays}

Of the 26 samples subjected to the 5'-UTR-VP2 screen, amplicons were recovered from 11 . The remaining 15 samples were negative for the screen. For the three VP1 snPCR screens, 19, 4 and 18 of the 26 samples subjected to these assays were positive for the PE, EVA/C and EV-B assays, respectively. (Table 1).

\section{Sequencing and identification}

All the 11 amplicons recovered from the $5^{\prime}$-UTR-VP2 screen were sequenced. However, only nine (9) of the sequence data generated were useable. The remaining two (2) were not useable due to the presence of multiple peaks. A BLASTn search of GenBank typed

Table 1. Results of the different PCR assays and identities of isolates typed.

\begin{tabular}{|c|c|c|c|c|c|c|c|c|c|c|}
\hline \multirow[b]{3}{*}{$\mathrm{S} / \mathrm{N}$} & & & \multicolumn{6}{|c|}{ VP1 ASSAYS } & \multirow[b]{3}{*}{ Serotypes } & \multirow[b]{3}{*}{ Number of Isolates } \\
\hline & \multicolumn{2}{|c|}{$\begin{array}{c}5^{\prime}-U T R-V P 2 \\
\text { ASSAY }\end{array}$} & \multicolumn{2}{|c|}{ AN89 (PE) } & \multicolumn{2}{|c|}{189 (EV-A/C) } & \multicolumn{2}{|c|}{187 (EV-B) } & & \\
\hline & PCR & SEQ & PCR & SEQ & PCR & SEQ & PCR & SEQ & & \\
\hline $\begin{array}{l}1 \\
2\end{array}$ & + & E24 & + & E24 & & & + & E24 & E24 & 1 \\
\hline \multicolumn{10}{|c|}{ 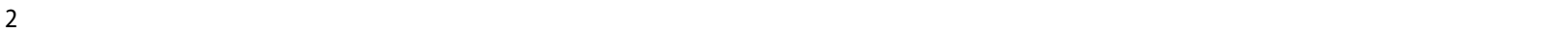 } & 1 \\
\hline 4 & & L 24 & + & CVB3 & & & & L 24 & CVB3 & 1 \\
\hline 5 & + & E19 & + & E19 & & & + & E19 & E19 & 1 \\
\hline 6 & + & E5 & + & E5 & & & + & E5 & E5 & 1 \\
\hline 7 & & & + & E5 & & & + & E5 & E5 & 1 \\
\hline 8 & & & + & CVB3 & & & $+W$ & UnD & CVB3 & 1 \\
\hline $9 \$$ & + & CVB3 & & & & & & & CVB3 & 1 \\
\hline 10 & + & E20 & + & E20 & + & $\mathrm{E} 20$ & + & E20 & E20 & 1 \\
\hline 11 & + & CVB5 & + & CVB5 & & & $+W$ & CVB5 & CVB5 & 1 \\
\hline 12 & & & + & CVB3 & & & $+W$ & CVB3 & CVB3 & 1 \\
\hline 13 & & & + & CVB3 & & & + & CVB3 & CVB3 & 1 \\
\hline 14 & & & & & & & $+W$ & CVB3 & CVB3 & 1 \\
\hline 15 & + & CVB3 & + & CVB3 & & & + & CVB3 & CVB3 & 1 \\
\hline 16 & $+W$ & UnD & & & & & & & & \\
\hline 17 & & & + & E11 & & & $+W$ & UnD & E11 & 1 \\
\hline $18 \$$ & + & EVB75 & & & & & $+W$ & UnD & EVB75 & 1 \\
\hline 19 & & & + & NS* & + & UnD & & & & \\
\hline 20 & & & + & UnD & & & $+W$ & UnD & & \\
\hline 21 & & & + & EVC99 & + & EVC99 & & & EVC99 & 1 \\
\hline \multicolumn{11}{|l|}{22} \\
\hline 23 & & & + & EVC99 & & & + & E11 & EVC99/E11 & 2 \\
\hline \multicolumn{11}{|c|}{ 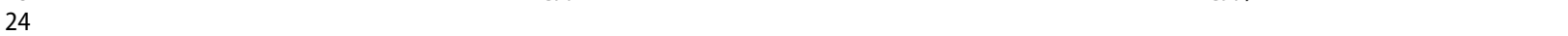 } \\
\hline 25 & & & + & E11 & & & + & E13 & E11/E13 & 2 \\
\hline \multirow[t]{2}{*}{26} & $+W$ & UnD & + & E13 & + & EVA71 & + & $N S^{*}$ & E13/EVA71 & 2 \\
\hline & 11 & 9 & 19 & 17 & 4 & 3 & 18 & 13 & & 23 \\
\hline
\end{tabular}

$\mathrm{W}=$ Weak; UnD = Unusable Data; E = Echovirus; EV = Enterovirus; CV = Coxsackievirus; NS* = Not Sequenced; $\$=$ Identified by only the $5^{\prime}$-UTR-VP2 assay NOTE: For all samples $(1,3,5,6,10,11$ and 15) that were simultaneously identified using both the 5'-UTR-VP2 and VP1 assays, the identities (EV type) of the samples as determined by both assays were $100 \%$ congruent. 
Table 2. Identification of enterovirus types using the 5'-UTR-VP2 sequence data and a BLASTn search of GenBank.

\begin{tabular}{ccccccc}
\hline S/N & Sample ID & Accession Number of Most Similar Sequence & Identity & E Value & Query Cover & Enterovirus Type \\
\hline 1 & 1 & KP036484.1 & $86 \%$ & 0.0 & $99 \%$ & E24 \\
2 & 3 & KP036484.1 & $86 \%$ & 0.0 & $99 \%$ & E24 \\
3 & 5 & KY792585.1 & $85 \%$ & 0.0 & $100 \%$ & E19 \\
4 & 6 & HM775882.1 & $82 \%$ & $9 \mathrm{e}-174$ & $100 \%$ & E5 \\
5 & 9 & KC481610.1 & $83 \%$ & $1 \mathrm{e}-172$ & $99 \%$ & CVB3 \\
6 & 10 & KF812551.1 & $87 \%$ & 0.0 & $100 \%$ & E20 \\
7 & 11 & KT285014.1 & $88 \%$ & 0.0 & $100 \%$ & CVB5 \\
8 & 15 & KR107057.1 & $85 \%$ & 0.0 & $100 \%$ & CVB3 \\
9 & 18 & AY556070.1 & $86 \%$ & 0.0 & $100 \%$ & EVB75 \\
\hline
\end{tabular}

the EVs as Echovirus (E) 5 (1 strain), E19 (1 strain), E20 (1 strain), E24 (2 strains), Coxsackievirus (CV) B3 (2 strains), CVB5 (1 strain) and EVB75 (1 strain) (Table 2).

Only 18 of the 19 amplicons recovered from the PE screen were sequenced. The 19th amplicon was not sequenced because, it leaked during transit (from Nigeria to South Korea) to the sequencing facility. Of the 18 amplicons sequenced, 17 were useable. The remaining one (1) was not useable due to the presence of multiple peaks. The Enterovirus Genotyping Tool (EGT) identified the 17 as E5 (2 strains), E11 (2 strains), E13 (1 strain), E19 (1 strain), E20 (1 strain), E24 (2 strains), Coxsackievirus (CV) B3 (5 strains), CVB5 (1 strain) and EVC99 (2 strains) (Table 1).

All the four (4) amplicons recovered from the EV-A/ $C$ screen were sequenced. However, three (3) of the sequence data generated were useable. The remaining one (1) was not useable due to the presence of multiple peaks. The EGT identified the three as E20 (1 strain), EVC99 (1 strain) and EVA71 genotype C1 (1 strain) (Table 1$)$.

Only 17 of the 18 amplicons recovered from the EV-B screen were sequenced. The 18th amplicon was not sequenced because, it leaked during transit (from Nigeria to South Korea) to the sequencing facility. Of the 17 amplicons sequenced, 13 were useable. The remaining four (4) were not useable due to the presence of multiple peaks. The Enterovirus Genotyping Tool (EGT) identified the 13 as E5 (2 strains), E11 (1 strain), E13 (1 strain), E19 (1 strain), E20 (1 strain), E24 (2 strains), Coxsackievirus (CV) B3 (4 strains) and CVB5 (1 strain) (Table 1).

In all, 20 of the 26 samples were identified as belonging to 11 (one EV-A, nine EV-Bs and one EVC) EV types. Also, 23 different enterovirus strains were identified in the 20 samples typed. Precisely, the 23 different enterovirus strains were EVA71 genotype C1 (1 strain), CVB3 (7 strains), CVB5 (1 strain), E5 (2 strains), E11 (3 strains), E13 (2 strains), E19 (1 strain), E20 (1 strain), E24 (2 strains), EVB75 (1 strain) and EVC99 (2 strains) (Table 3).

\section{Comparison of the 5'-UTR-VP2 and the VP1 screens}

Of the 11 EV types identified in this study, the 5'UTR-VP2 assay was able to identify seven (7) of them while the VP1 assay identified 10 of them (Table 3). Both assays simultaneously detected seven (7) of the $11 \mathrm{EV}$ types identified in this study (Table 1). EVB75 was only identified by the 5'UTR-VP2 assay while E11, E13, EVA71 and EVC99 were only identified by the VP1 assay (Table 3 ).

\section{Phylogenetic analysis}

Only the EVA71 described in this study was subjected to phylogenetic analysis because of the association of EVA71 (and especially Genotype C strains) with severe neurological manifestations [19]. The tree (Figure 2) confirms that the EVA71 described in this study belongs to genotype $\mathrm{C} 1$. It further confirms (though with poor bootstrap support) the result of a BLASTn search (data not shown) that showed the EVA71 described in this study is most similar to that detected in a child with AFP in Cameroon in 2008 [20].

Table 3. Enterovirus types identified in this study, the genomic region used for identification and number of isolates of each type.

\begin{tabular}{|c|c|c|c|c|c|}
\hline \multirow[b]{2}{*}{$\mathrm{S} / \mathrm{N}$} & \multirow[b]{2}{*}{$\begin{array}{l}\text { EV } \\
\text { Type }\end{array}$} & \multicolumn{2}{|c|}{ Region Identified } & \multirow{2}{*}{$\begin{array}{c}\text { Number } \\
\text { of } \\
\text { Isolates }\end{array}$} & \multirow[b]{2}{*}{$\begin{array}{c}\text { Cummulative } \\
\text { Number }\end{array}$} \\
\hline & & 5'-UTR & VP1 & & \\
\hline 1 & $\mathrm{CVB}^{5}$ & + & + & 7 & 7 \\
\hline 2 & CVB5 & + & + & 1 & 8 \\
\hline 3 & E5 & + & + & 2 & 10 \\
\hline 4 & E11 & & + & 3 & 13 \\
\hline 5 & E13 & & + & 2 & 15 \\
\hline 6 & E19 & + & + & 1 & 16 \\
\hline 7 & E20 & + & + & 1 & 17 \\
\hline 8 & E24 & + & + & 2 & 19 \\
\hline 9 & EVB75* & + & & 1 & 20 \\
\hline 10 & EVA71 & & + & 1 & 21 \\
\hline \multirow[t]{2}{*}{11} & EVC99 & & + & 2 & 23 \\
\hline & & & & 23 & \\
\hline
\end{tabular}

* = Identified by only the $5^{\prime}$-UTR - VP2 assay; ${ }^{5}=$ one of the isolates was Identified by only the 5'-UTR - VP2 assay 


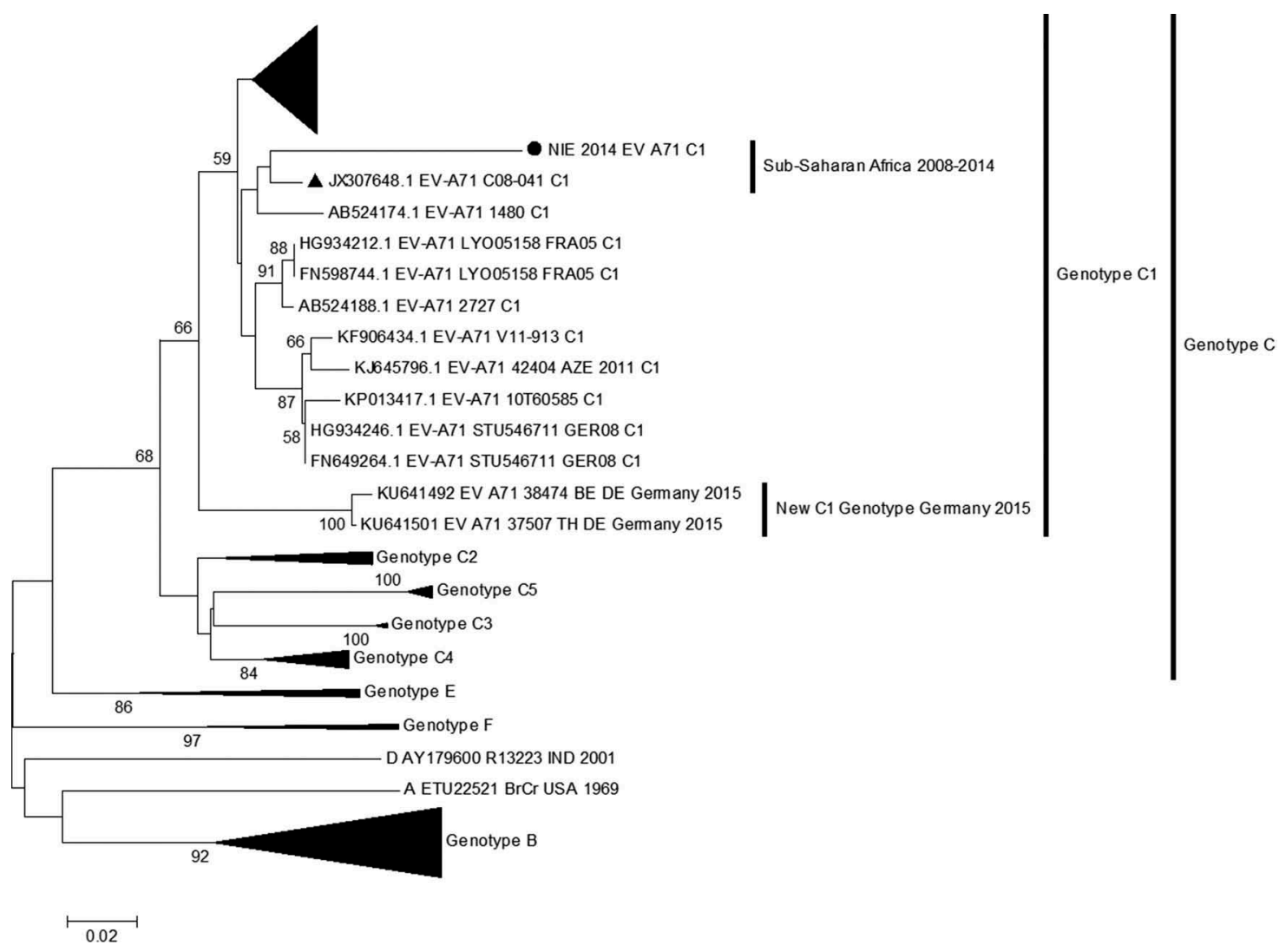

Figure 2. Phylogram of genetic relationship between VP1 nucleotide sequences of EV-A71.The phylogenetic tree is based on an alignment of the partial VP1 sequences. The reference EVA71 sequences described in Bessaud et al. [21], was used as baseline. Added to it was the EVA71 described in this study alongside the top 50 hits of a BLASTn search using the EVA71 described in this study as query. We also added two reference sequences representing the new C1 lineage first described in Germany in 2015 by Böttcher et al. [22]. The newly sequenced strain is indicated with black circle. The only other genotype $\mathrm{C} 1$ strain detected and described in Africa till date is indicated with a black triangle. Bootstrap values are indicated if $>50 \%$.

\section{Discussion}

In our 2014 study [11], there were 27 isolates recovered on RD cell line from AFP cases that we could not determine their serotypes. This study was aimed at using alternative strategies to determine the serotypes of these previously untypables isolates. At the commencement of this study we had access to 26 of the samples (the 27th was already exhausted). We successfully identified 20 of the 26 samples analyzed. From the 20 samples, 23 (three samples had co- or mixed infection) EV strains were recovered, 20 of which were EV-Bs (Table 1). This is not surprising in the light of the EV-B bias of RD cell line [20, 22-24].

Of the 23 EV strains typed in this study, 21 were unambiguously identified using the VP1 assay (Table 1). As shown in Figure 1, two basic differences exist between the VP1 assay used in this study and that used in Faleye et al. [11]. The first difference lies in the primers used for cDNA synthesis. As opposed to the previous study [11] where CDNA was done using Random Hexamers, cDNA for the VP1 assay was made using the primers AN32-AN35 $[6,12]$. This obviously, significantly improved our capacity to identify some of the previously untypable EVs. In fact, 17 of the 21 strains detected by the VP1 assay can be accounted for by this modification in the assay design. This therefore confirms, the sensitivity of this cDNA synthesis algorithm as initially designed by Nix et al. [12], and its consequent superiority to using random Hexamers for EV VP1 amplification.

The second difference between the VP1 assay used in this study and that used in Faleye et al. [11], lies in the second round PCR assays. While only one second round PCR assay was done in Faleye et al. [11], three different second round PCR assays were done in this study (Figure 1). We had previously shown that this modification increases the sensitivity of the assay and especially its capacity for EV co-infection resolution $[1516,24,25]$. In this study, four (4) of the 21 strains detected by the VP1 assay can be accounted for by this modification in the assay design. Of the four strains detected exclusively by this modification, the CVB3 in Sample 14 (Table 1) would have been completely missed if not for this assay modification. This shows that a sample declared negative by the AN89-AN88 (PE) assay might not be truly negative. Considering the very variable nature of the VP1 protein, it is possible that the virus in the sample of interest might have mutations in the AN89 primer 
binding site that makes it impossible for the primer to bind to the site.

The other three strains detected exclusively by this modification (three different second round PCR assays; Figure 1) were cases of co-infection (Table 1). To be precise, the E11, E13 and EVA71-C1 detected in samples 23, 25 and 26 would have also been missed should we have relied on the PE assay alone for EV VP1 amplification. Considering how common EV coinfection is (3/26; in this study) [25], coupled with their role in CVDPV emergence [26,27], we have repeatedly $[13,15,25,26]$ emphasized the need to employ co-infection resolution assays when prospecting for enteroviruses. Against this backdrop, it is important to bear in mind that any EV surveillance study that uses the PE (AN89 + AN88) assay alone for EV VP1 detection and subsequently, EV identification might not be capturing the complete picture of EV diversity in the samples screened.

This modified protocol enabled us to detect the EVA71 genotype $\mathrm{C} 1$ for the first time in Nigeria. Prior this study, only genotype E of EVA71 had been described in the country $[10,30]$. Both a BLASTn search (data not shown) of the GenBank and phylogenetic analysis (Figure 2) suggests that the EVA71 genotype C1 described in this study is most similar to one recovered from a child with AFP in Cameroon in 2008 [20]. Though EVA71 genotype Cs have been associated with clinical manifestations that might have neurological complication, and both EVA71 genotype $\mathrm{C} 1$ strains described in sub-Saharan Africa till date were from children with AFP, it might be premature to conclude they were responsible for the associated clinical presentation. For example, firstly, in the Nigeria case described in this study, if not for the co-infection resolution modification to the VP1 assay, only E13 would have been detected in that isolate. In the light of co-infection with both E13 and EVA71 genotype C1 in this case, to which of the two EV types do we ascribe the clinical presentation? Of course, the synergistic effect of EV coinfections cannot be overlooked, but until rigorous experimental data is provided to document such associations, it is only reasonable that all be interpreted with caution. Especially those cases in which no attempt was made to detect or resolve possible co-infections.

Furthermore, coupled with the results of the BLASTn search (data not shown), the topology (though with poor bootstrap support) of the EVA71 tree suggest that the clade detected in Cameroon in 2008 and subsequently in Nigeria in 2014 might have been circulating between that time (and if the regional confinement hypothesis is anything to go by) in the region. Considering that as at the time of writing there were not many EV-A71 genotype C1 from sub-Saharan Africa in GenBank, we do not think it unusual to have such finding. Whether this clade is still present and circulating in the region is not known. However, this finding further necessitates the need to rigorously search for the virus in the population. What is certain is that the subSaharan Africa EV-A71 C1 strain described in this study, belongs to the old $\mathrm{C} 1$ lineage and not the new lineage first described in Germany in 2015 [22].

With respect to the 5'-UTR-VP2 assay, of the 23 EV strains typed in this study, 9 were identified using this assay (Table 2). It is however important to note that seven (7) of the nine (9) EV types identified by the 5'-UTR-VP2 assay were also simultaneously identified by the VP1 assays and their identities by both assays were congruent (Table 1). The remaining two isolates only identified using the results of the 5'-UTR-VP2 assay was because the VP1 assays failed to work on these isolates. Hence, there was no VP1 data to compare (Tables 1 and 3). However, considering the $100 \%$ congruence of those for which both $5^{\prime}$ UTR-VP2 and VP1 data exist (Table 1), we feel comfortable taking the identification as correct as several other studies have shown $[14,29]$ and was recently recommended by the European Non-polio Enterovirus Network (ENPEN) [30]. Our findings therefore also demonstrate the usefulness of this assay for EV identification.

\section{Conclusion}

In this study we identified 20 of 26 samples that were previously untypable [11]. We showed that modifying the algorithm used in Faleye et al., 2017 to include AN32-AN35 specific cDNA synthesis and expanding the second round PCR assay to accommodate coinfection resolution can significantly increase the sensitivity of the EV VP1 assay. We further provided evidence that suggest that a clade of EVA71 genotype C1 might have been circulating in sub-Saharan Africa since 2008. Finally, we showed that the 5'-UTR-VP2 assay might be as valuable as the VP1 assay in EV identification.

\section{Acknowledgments}

We thank the WHO National Polio Laboratory in Ibadan, Nigeria for providing the anonymous isolates analyzed in this study. This study was funded by contributions from authors.

\section{Author Contributions}

1. Study Design (AMO, FTOC, AJA)

2. Sample Collection, laboratory and data analysis (All Authors)

3. Wrote the first draft of the Manuscript (AMO and FTOC)

4. Revised the Manuscript (All Authors)

5. Read and Approved the Final Draft (All Authors)

6. AJA supervised this study

\section{Data availability statement}

The Sequence data that support the findings of this study are openly available in GenBank (https://www.ncbi.nlm.nih. 
gov/genbank/) with accession numbers MH379115-MH 379135 and MH397260-MH397268.

\section{Disclosure statement}

No potential conflict of interest was reported by the authors.

\section{ORCID}

Christopher Olaoluwa Okeowo (D) http://orcid.org/00000002-8407-9080

\section{References}

[1] http://www.picornaviridae.com/enterovirus/entero virus. Last accessed November 19, 2018.

[2] World Health Organisation. Guidelines for environmental surveillance of poliovirus circulation. Switzerland: Geneva; 2003.

[3] World Health Organisation. Polio laboratory manual. 4th ed. Switzerland: Geneva; 2004.

[4] Oberste MS, Maher K, Williams AJ, et al. Speciesspecific RT-PCR amplification of human enteroviruses: a tool for rapid species identification of uncharacterized enteroviruses. J Gen Virol. 2006;87:119-128.

[5] Oberste MS, Maher K, Kilpatrick DR, et al. Molecular evolution of the human enteroviruses: correlation of serotype with VP1 sequence and application to picornavirus classification. J Virol. 1999;73(3):1941-1948.

[6] World Health Organisation. Enterovirus surveillance guidelines: guidelines for enterovirus surveillance in support of the polio eradication initiative. Switzerland: Geneva; 2015.

[7] McAllister RM, Melnyk J, Finklestein JZ, et al. Cultivation in vitro of cells derived from a human rhabdomyosarcoma. Cancer. 1959;24:520-526.

[8] Pipkin PA, Wood DJ, Racaniello VR, et al. Characterization of $L$ cells expressing the human poliovirus receptor for the specific detection of polioviruses in vitro. J Virol Methods. 1993;41:333-340.

[9] Yoshii K, Yoneyama T, Shimizu H, et al. Sensitivity of cells to poliovirus. Jpn J Infect Dis. 1999;52:169.

[10] Oyero OG, Adu FD, Ayukekbong JA. Molecular characterization of diverse species enterovirus-B types from children with acute flaccid paralysis and asymptomatic children in Nigeria. Virus Res. 2014;189:189-193.

[11] Faleye TOC, Adewumi MO, Japhet MO, et al. Non-polio enteroviruses in faeces of children diagnosed with acute flaccid paralysis in Nigeria. BMC Virol J. 2017;14:175.

[12] Nix WA, Oberste MS, Pallansch MA. Sensitive, seminested PCR amplification of VP1 sequences for direct identification of all enterovirus serotypes from original clinical specimens. J Clin Microbiol. 2006;44(8):2698-2704.

[13] Faleye TOC, Adewumi MO, Ozegbe NP, et al. Extending the utility of the WHO recommended assay for direct detection of enteroviruses from clinical specimen for resolving poliovirus co-infection. BMC Res Notes. 2018;11(1):47.

[14] Kubo H, Iritani N, Seto Y. Molecular classification of enteroviruses not identified by neutralization tests. Emerg Infect Dis. 2002;8(3):298-304.

[15] Adeniji JA, Oragwa AO, George UE, et al. Preponderance of enterovirus species C in RD-L20B cell culture negative stool samples from children diagnosed with Acute Flaccid Paralysis in Nigeria. Arch Virol. 2017a;162(10):3089-3101.

[16] Kroneman A, Vennema H, Deforche K, et al. An automated genotyping tool for enteroviruses and noroviruses. J Clin Virol. 2011;51:121-125.

[17] Tamura K, Peterson D, Peterson N, et al. MEGA5: molecular evolutionary genetics analysis using maximum likelihood, evolutionary distance, and maximum parsimony methods. Mol Biol Evol. 2011;28(10):2731-2739.

[18] Kimura M. A simple method for estimating evolutionary rate of base substitutions through comparative studies of nucleotide sequences. J Mol Evol. 1980;16(2):111-120.

[19] Tao J, He XY, Shi Y, et al. epidemiology of 45,616 suspect cases of hand, foot and mouth disease in Chongqing, China, 2011-2015. Sci Rep. 2017;7:45630.

[20] Sadeuh-Mba SA, Bessaud M, Massenet D, et al. High frequency and diversity of species $C$ enteroviruses in Cameroon and neighbouring countries. J Clin Microbiol. 2013;51(3):759-770.

[21] Bessaud M, Razafindratsimandresy R, Nougaire 'De A, et al. Molecular comparison and evolutionary analyses of VP1 nucleotide sequences of New African human enterovirus 71 isolates reveal a wide genetic diversity. PLoS One. 2014;9(3):e90624.

[22] Böttcher S, Obermeier PE, Neubauer K, et al. Recombinant enterovirus A71 subgenogroup C1 strains, Germany, 2015. Emerg Infect Dis. 2016;22(10):1843-1846.

[23] Faleye TOC, Adeniji JA. Enterovirus species B bias of RD cell line and its influence on enterovirus diversity landscape. Food Environ Virol. 2015;7(4):390-402.

[24] Majumdar M, Martin J. Detection by direct next generation sequencing analysis of emerging enterovirus D68 and C109 strains in an environmental sample from Scotland. Front Microbiol. 2018;9:1956.

[25] Adeniji JA, Ayeni FA, Ibrahim A, et al. Comparison of algorithms for the detection of enteroviruses in stool specimens from children diagnosed with Acute Flaccid Paralysis. J Pathog. 2017b;9. Article ID 9256056. DOI:10.1155/2017/9256056

[26] Faleye TOC, Adewumi MO, Kareem SA, et al. The Impact of a panenterovirus Vp1 assay on our perception of the enterovirus diversity landscape of a sample. J Hum Virol Retrovirol. 2016a;4(3):00134.

[27] Rao CD, Yergolkar P, Shankarappa KS. Antigenic diversity of enteroviruses associated with nonpolio acute flaccid paralysis, India, 2007-2009. Emerg Infect Dis. 2012;18 (11):1833-1840.

[28] Combelas N, Holmblat B, Joffret M, et al. Recombination between poliovirus and coxsackie a viruses of species $C$ : a model of viral genetic plasticity and emergence. Viruses. 2011;3:1460-1484.

[29] Burns CC, Shaw J, Jorba J, et al. Multiple independent emergences of type 2 vaccine-derived polioviruses during a large outbreak in Northern Nigeria. J Virol. 2013;87:4907-4922.

[30] Faleye TOC, Adewumi MO, Coker BA, et al. Direct detection and identification of enteroviruses from faeces of healthy Nigerian children using a cell-culture independent RT- semi-nested PCR assay. Adv Virol. 2016b. DOI:10.1155/2016/1412838.

[31] Chu PY, Lin KH, Hwang KP, et al. Molecular epidemiology of enterovirus 71 in Taiwan. Arch Virol. 2001;146:589-600.

[32] Harvala H, Broberg E, Benschop $K$, et al. Recommendations for enterovirus diagnostics and characterisation within and beyond Europe. J Clin Virol. 2018;101:11-17. 